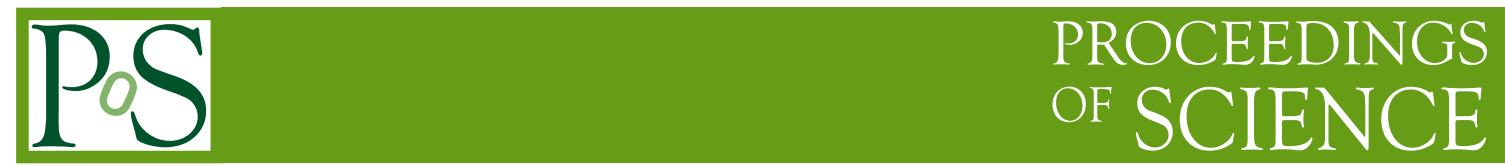

\title{
Current status of nPDFs and future facilities
}

\author{
Carlota Andres* \\ Instituto Galego de Física de Altas Enerxías IGFAE, Universidade de Santiago de Compostela, \\ E-15782 Santiago de Compostela (Galicia-Spain) \\ E-mail: carlota.andrescusc.es
}

In this talk, the current status of global analyses in QCD of collinearly factorized nuclear parton distribution functions, nPDFs, is reviewed. The most recent nPDF sets available are presented and compared, focusing on the latest improvements and the next steps to be made. Finally, the potential to constrain the partonic structure of the nuclei in future facilities, such as the Electron Ion Collider (EIC), is outlined.

QCD Evolution 2017

22-26 May, 2017

Jefferson Lab, Newport News, VA - USA

${ }^{*}$ Speaker. 


\section{Introduction}

Nuclear parton distribution functions (nPDFs) are essential for the analysis of a wide variety of nuclear experiments, such as heavy ion collisions at the Large Hadron Collider (LHC) and at the Relativistic Heavy-Ion Collider (RHIC). They are also of interest for the proton community, as free proton PDFs global fits make use of neutrino and deuterium DIS experiments, where nuclear modifications have to be taken into account. Furthermore, the precise knowledge of nPDFs will be mandatory for future colliders, like the EIC and the LHeC. In the last few years, there has been a lot of improvement on the determination of nPDFs, both from the experimental and the theoretical sides, which will be summarized in the following sections.

The standard technique to obtain (nuclear) PDFs is called global analysis. This is founded on collinear factorization $[1,2]^{1}$ : in hard processes different energy scales arise, allowing the cross section to be computed at the parton level using pQCD, $d \hat{\sigma}_{i j}\left(\mu_{r}^{2}, \mu_{f}^{2}\right)$, and then convoluted with the large distance information about the structure of the initial state hadrons, that is, the nonperturbative parton distribution functions, $f_{i}\left(x_{i}, \mu_{f}^{2}\right)$,

$$
d \sigma=\sum_{i, j} f_{i}\left(x_{i}, \mu_{f}^{2}\right) \otimes d \hat{\sigma}_{i j}\left(\mu_{r}^{2}, \mu_{f}^{2}\right) \otimes f_{j}\left(x_{j}, \mu_{f}^{2}\right)+\mathscr{O}\left(\mu_{f}^{2 n}\right) .
$$

.eps Although the computation of the parton distribution functions is beyond the scope of pQCD, their dependence on the factorization scale, $\mu_{f}$, is perturbative and it is given by the well-known DGLAP evolution equations $[4,5,6]$. Because of their universality, (n)PDFs can be extracted directly from various experiments (deep inelastic scattering (DIS), Drell-Yan (DY), etc.) through global analyses.

\section{The technique}

In general lines a global fit can be summarized as follows: First, the data to be fitted are selected. Then, the PDFs are parametrized at the initial scale, $Q_{0}$, and evolved - using DGLAP equations - from this initial scale to that of the experiment, $Q>Q_{0}{ }^{2}$ At this stage and thanks to factorization, the theoretical observable (usually a cross section or a structure function) can be computed. Finally, a $\chi^{2}$-distribution is built and the value of the parameters is found iteratively minimizing the $\chi^{2}$.

So far in this text, all the formalism presented was valid both for free parton distribution functions (PDFs) and parton distribution functions in nuclear media (nPDFs). However, are the nuclear bounding effects in nPDFs significant? Since 1982, thanks to the European Muon Collaboration data on $F^{F e} / F^{d}$ [10], we know that nuclear effects cannot be neglected. As most of the heavy nuclei data used in nPDFs analyses are ratios over the proton or deuteron measurement, in most of the nPDFs global fits, instead of prametrizing the nPDFs themselves, the parametrized quantities are the so-called nuclear modifications, $R_{i}^{A}\left(x, Q^{2}\right)$,

$$
f_{i}^{p / A}\left(X, Q_{0}^{2}\right)=R_{i}^{A}\left(x, Q_{0}^{2}\right) f_{i}^{p}\left(x, Q_{0}^{2}\right),
$$

\footnotetext{
${ }^{1}$ Factorization has not been formally proven for nuclear media, but, for instance, in heavy ion collisions is a standard assumption supported by numerous studies [3].

${ }^{2}$ Several techniques to accomplish a numerical solution of these integro-differential equations have been developed both in $x$-space [7, 8], and Mellin space [9].
} 
where $f_{i}^{p / A}\left(x, Q_{0}^{2}\right)$ and $f_{i}^{p}\left(x, Q_{0}^{2}\right)$ are, respectively, the bound proton and the free proton PDFs. The nuclear PDFs, which are those entering in the factorization formula, Eq. (1.1), are built as a linear combination of the bound proton, $f_{i}^{p / A}$, and bound neutron, $f_{i}^{n / A}$, PDFs as follows,

$$
f_{i}^{A}\left(X, Q^{2}\right)=\frac{Z}{A} f_{i}^{p / A}\left(X, Q^{2}\right)+\frac{A-Z}{A} f_{i}^{n / A}\left(X, Q^{2}\right),
$$

where $Z$ and $A$ are, respectively, the atomic and mass numbers.

Due to the lack of diversity of the experimental data available, nuclear modifications for each parton flavor cannot be independently determined. Therefore, most of the analyses, as EPS09 [11], DSSZ12 [12], and KA15 [13], imposed flavor independence of the valence and light sea quarks,

$$
R_{u_{v}}^{A}\left(x, Q_{0}^{2}\right)=R_{d_{v}}^{A}\left(x, Q_{0}^{2}\right) \quad R_{\bar{u}}^{A}\left(x, Q_{0}^{2}\right)=R_{\bar{d}}^{A}\left(x, Q_{0}^{2}\right)=R_{\bar{s}}^{A}\left(x, Q_{0}^{2}\right) .
$$

This assumption turned out to be consistent with data but it has no theoretical support, as DGLAP evolution destroys flavor independence. Some of the most recent analyses have begun to incorporate flavor separation: nCTEQ15 [14] for the valence quarks, and EPPS16 [15] for both valence and light sea quarks.

\section{Comparison of the current nPDF sets}

Recently, the first global fit of nPDFs which includes LHC p+Pb Run-I data, EPPS16[15], appeared, but let us start first where we were before the first run of the LHC.

All the sets available include neutral current DIS and Drell-Yan data. Nowadays, the available charged-lepton DIS measurements go from $x \approx 0.008$ to $x \approx 1$. These data are basically sensitive to the valence quarks, though for $x \leq 0.01$ some sensitivity to the sea quarks arises. Gluons are almost not constrained by DIS and DY experiments. Many analyses such as EPS09, DSSZ12, nCTEQ15, and the recent EPPS16, have also included among their data inclusive pion production in $\mathrm{d}$-Au collisions at RHIC, since this observable may have an impact on constraining the gluon distribution. Nevertheless, inclusive pion data have an additional dependence on the fragmentation functions (FFs), consequently, their interpretation is still ambiguous. With respect to neutrino DIS experiments, they may be helpful to constrain light quark flavor, since they provide an electroweak observable, however, they have only been employed in DSSZ12 and EPPS16 fits.

In Fig. 1 the nuclear modification factors of the lead nuclei at $Q^{2}=10 \mathrm{GeV}^{2}$ versus $x$ for different nPDF sets are presented. The areas with yellow background are those where there are no direct constraints from experimental data. Regarding the nuclear modification for the up valence and the light sea quarks, all the available sets show agreement in the data-constrained region ${ }^{3}$. None of the sets shown include any flavor separation nor for the valence neither for the light sea quarks. At low- $x$ there are no data constraints at all, therefore, the results are extrapolations which are not reliable since they depend on the initial parametrization used by the group - which varies from one collaboration to another. Since DIS and DY data are almost insensitive to the gluon, this is the less known nPDF. In fact, as it can be seen in Fig. 1, HKN07, EPS09, and DSSZ12 analyses exhibit large differences in the nuclear modification of the gluon. As it was already mentioned,

\footnotetext{
${ }^{3}$ These results are also compatible with those of nCTEQ15 which are not shown here.
} 
EPS09 and DSSZ12 employ inclusive pion production to try to constrain the gluon distribution, but still their results differ in the data-constrained region. The main reason for this disagreement is that EPS09 uses vacuum pion fragmentation functions and includes pion data with a weight, so they have more impact on the fit, while DSSZ makes use of medium-modified FFs. The result for the gluon of nCTEQ15 - not presented here - has larger uncertainties due, mainly, to the larger cuts that this collaboration applies to the data.

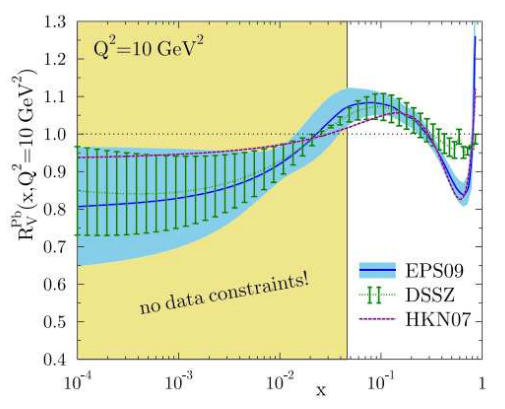

(a) Valence

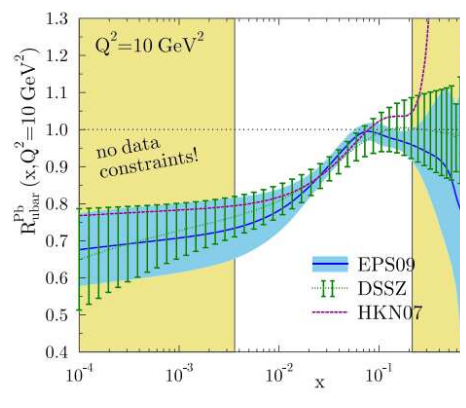

(b) Sea

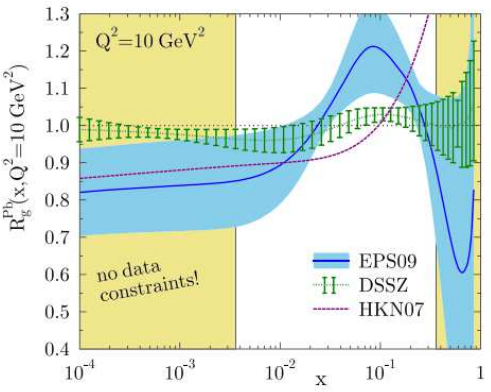

(c) Gluon

Figure 1: Comparison of the nuclear modifications of HKN07 (magenta), EPS09 (blue), and DSSZ (green) for up valence, sea quark, and gluon for $\mathrm{Pb}$ at $Q^{2}=10 \mathrm{GeV}^{2}$. Figure taken from [16].

To summarize, the nPDFs analyses available present considerable differences. To reduce these differences more data are needed. The LHC proton-lead measurements probe a totally distinct kinematical region corresponding to a much higher $Q^{2}$ and to an $x$-range from $x \approx 0.001$ to $x \approx 0.5$. Therefore, these data may play an important role to impose better constraints on the nPDFs. In fact, it was found, by means of the Bayesian reweighting technique, that these data could lead to a sizable reduction of the uncertainties on nuclear PDFs [17, 18].

As mentioned above, the only set of nPDFs which include any LHC p+Pb data is EPPS16. This fit incorporates both the electroweak boson production $[19,20]$ and the CMS dijet measurements in $\mathrm{p}+\mathrm{Pb}$ collisions [21]. The inclusion of the former had potential to analyze the flavor separation, although, due to the lack of statistics, these data did not show a substantial influence on the fit. Nevertheless, neutrino DIS measurements are employed in this analysis -absent in its predecessor, EPS09 - making possible the study of flavor separation of both valence and sea quarks. On the other hand, the dijet measurements have provided a better constrained nuclear modification of the gluon in the high- $x$ domain.

Let us compare now this new set with the previous ones. In Fig. 2 the valence nuclear modifications for the lead nuclei at $Q^{2}=10 \mathrm{GeV}^{2}$ for different nPDFs analyses are presented. In the leftmost and center panels of this figure, the results for the $u$ valence and $d$ valence, respectively, of nCTEQ15 and EPPS16 are shown. The results of both fits are compatible within their uncertainties. The nCTEQ15 uncertainties are, in general, considerably smaller than those of EPPS16, due to the fact that nCTEQ15 imposes more restrictions on the valence quarks than EPPS16. In order to compare to EPS09 and DSSZ12, since these demanded total flavor independence, EPPS16 
makes use of the flavor average,

$$
R_{V}^{A}=\frac{u_{v}^{p / P b}+d_{v}^{p / P b}}{u_{v}^{p}+d_{v}^{p}}
$$

as it is shown in the rightmost panel of Fig. 2. All the sets give compatible results, except for DSSZ12 in the EMC-effect region, which is likely due to a misinterpretation of the isospin corrections applied to the data by the experiments ${ }^{4}$. Finally, it is worth emphasizing that the employment of neutrino DIS data have allowed EPPS16 to consider flavor separation, but the results show that $R_{u_{v}}^{A} \sim R_{d_{v}}^{A}$.

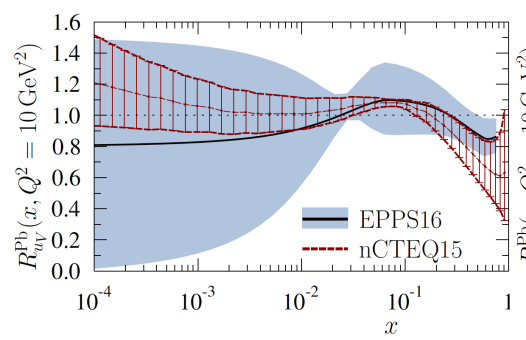

(a) $R_{u_{v}}^{A}$

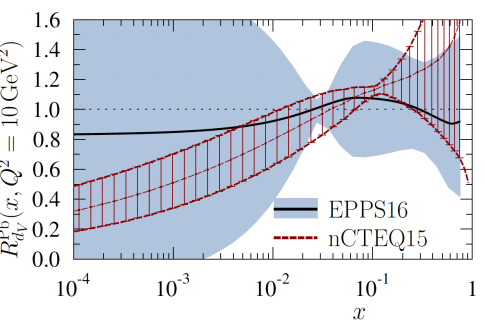

(b) $R_{d_{v}}^{A}$

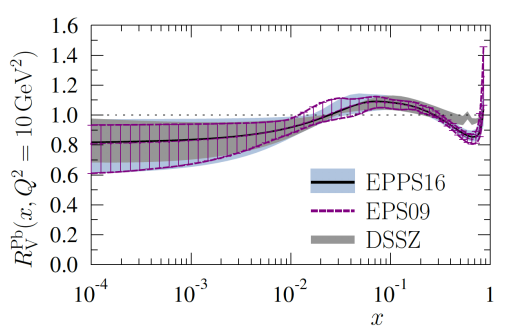

(c) $R_{V}^{A}$

Figure 2: Comparison of the valence nuclear modifications for lead at $Q^{2}=10 \mathrm{GeV}^{2}$. Leftmost and center panels: nCTEQ15 (dashed red), and EPPS16 (blue). Rightmost panel: EPS09 (purple), DSSZ12 (gray), and EPPS16 (blue). Figures taken from [15].

The sea nuclear modifications for the lead nuclei at $Q^{2}=10 \mathrm{GeV}^{2}$ are presented in Fig. 3 . The nCTEQ15 uncertainties for the light sea quarks are much smaller than those of EPPS16, since nCTEQ15 applies much more restrictive assumptions to the sea quarks than EPPS16. To compare EPPS16 sea nuclear modifications with those of EPS09 and DSSZ12, the flavor averaged sea nuclear modification is defined as,

$$
R_{S}^{A}=\frac{\bar{u}^{p / P b}+\bar{d}^{p / P b}+\bar{s}^{p / P b}}{\bar{u}^{p}+\bar{d}^{p}+\bar{s}^{p}} .
$$

In the rightmost panel of Fig. 3, it can be observed that the three fits give compatible results, being EPPS16 the one with larger uncertainties, but also the least biassed, since the sea quarks of EPPS16 have more degrees of freedom.

Fig. 4 shows the gluon nuclear modification factor for lead at $Q^{2}=10 \mathrm{GeV}^{2}$. As for the previous figures, the nCTEQ15 uncertainties are, generally, smaller than those of EPPS16, as the latter has more free parameters. Nonetheless, in the high- $x$ region the nCTEQ15 gluon shows larger uncertainties, since no LHC data are included in this fit - EPPS16 includes dijet measurements in $\mathrm{p}+\mathrm{Pb}-$ and since the cuts that nCTEQ15 applies to the DIS data are more limiting than those of EPPS16. The DSSZ12 gluon shows almost no nuclear modifications, due to the use in their fit of medium modified fragmentation functions. While EPPS16 includes more data - CMS dijet data which could help to constrain the gluon better than EPS09, its uncertainties are larger, due, mainly, to the most restrictive assumptions on the EPS09 fit.

\footnotetext{
${ }^{4}$ This matter can also be observed in the leftmost panel of Fig. 1
} 


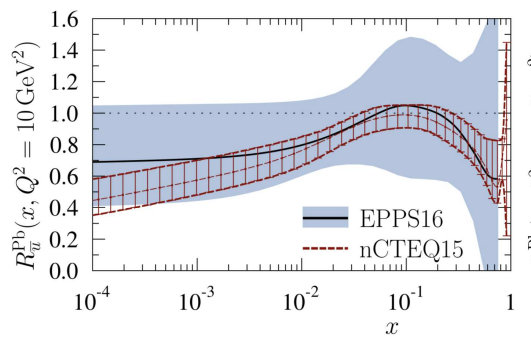

(a) $R_{\bar{u}}^{A}$

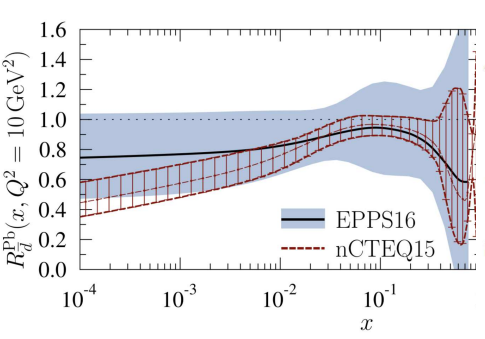

(b) $R_{\bar{d}}^{A}$

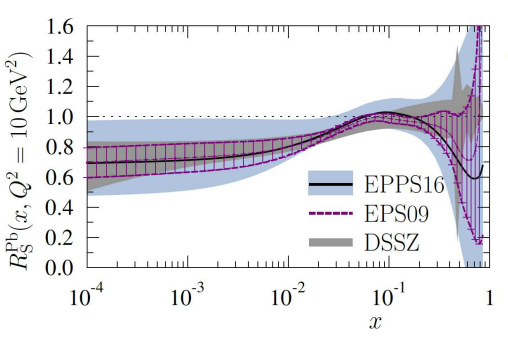

(c) $R_{S}^{A}$

Figure 3: Comparison of the light sea nuclear modifications for lead at $Q^{2}=10 \mathrm{GeV}^{2}$. Leftmost and center panels: nCTEQ15 (dashed red), and EPPS16 (blue). Rightmost panel: EPS09 (purple), DSSZ12 (gray), and EPPS16 (blue). Figures taken from [15].
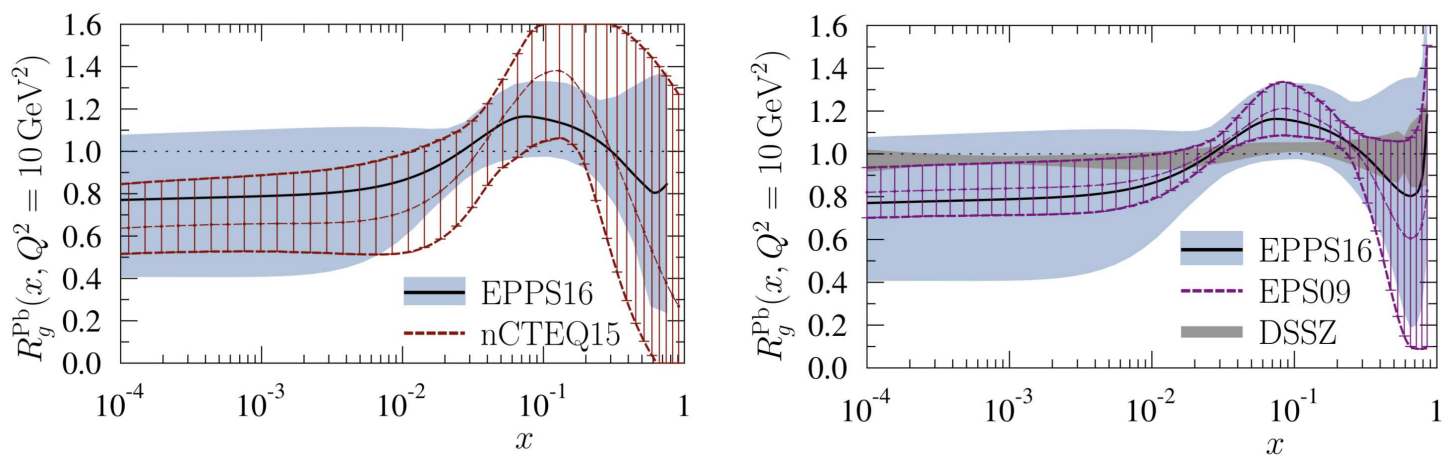

Figure 4: Comparison of the gluon nuclear modifications for lead at $Q^{2}=10 \mathrm{GeV}^{2}$. Leftmost and center panels: nCTEQ15 (dashed red), and EPPS16 (blue). Rightmost panels: EPS09 (purple), DSSZ12 (gray), and EPPS16 (blue). Figures taken from [15].

\section{Outlook}

The first proton-lead run at the LHC has opened a new kinematic region, giving rise to novel constraints to the nPDFs. Nevertheless, nPDFs are still much less known than free proton PDFs, especially due to the wider diversity of data that cover a larger kinematical range available for the proton community.

All the LHC $\mathrm{p}+\mathrm{Pb}$ data that were included in EPPS16, where backward-to-forward ratios in order to reduce the sensitivity to the chosen free-proton baseline PDFs. The p-p reference at $\sqrt{s_{\mathrm{NN}}}=5.02 \mathrm{TeV}-$ same center of mass energy as the $\mathrm{p}+\mathrm{Pb}$ data - is now available, making possible the inclusion of new $\mathrm{p}+\mathrm{Pb}$ observables in future fits and a cleaner sensitivity to the nuclear modifications. In addition, since the correlation on the systematics uncertainties are not provided by the experiments, all the uncertainties regarding the LHC data are added in quadrature in EPPS16. It would be convenient to take into account these correlations.

Moreover, the p-p reference for the LHC Run-II at $\sqrt{s_{\mathrm{NN}}}=8 \mathrm{TeV}$ is nowadays available. This would allow us to include other measurements, such as Drell-Yan at the forward direction at the $\mathrm{LHCb}$, which would provide a probe of light sea quarks at small- $x$ [22]. 
Nonetheless, LHC observables are limited to a relatively high- $Q^{2}$ domain, so, obtaining constraints at the low- $x$ and low- $Q^{2}$ kinematic region is troublesome. A new generation of DIS experiments is crucial in order to probe the nPDFs at low $x$. Two facilities have been proposed: the Electron-Ion Collider (EIC) [23] at the United States and the Large Hadron electron Collider (LHeC) [24] at CERN. The wide kinematic range that would cover the EIC and the LHeC are shown, respectively, on the left and right panels of Fig. 5.
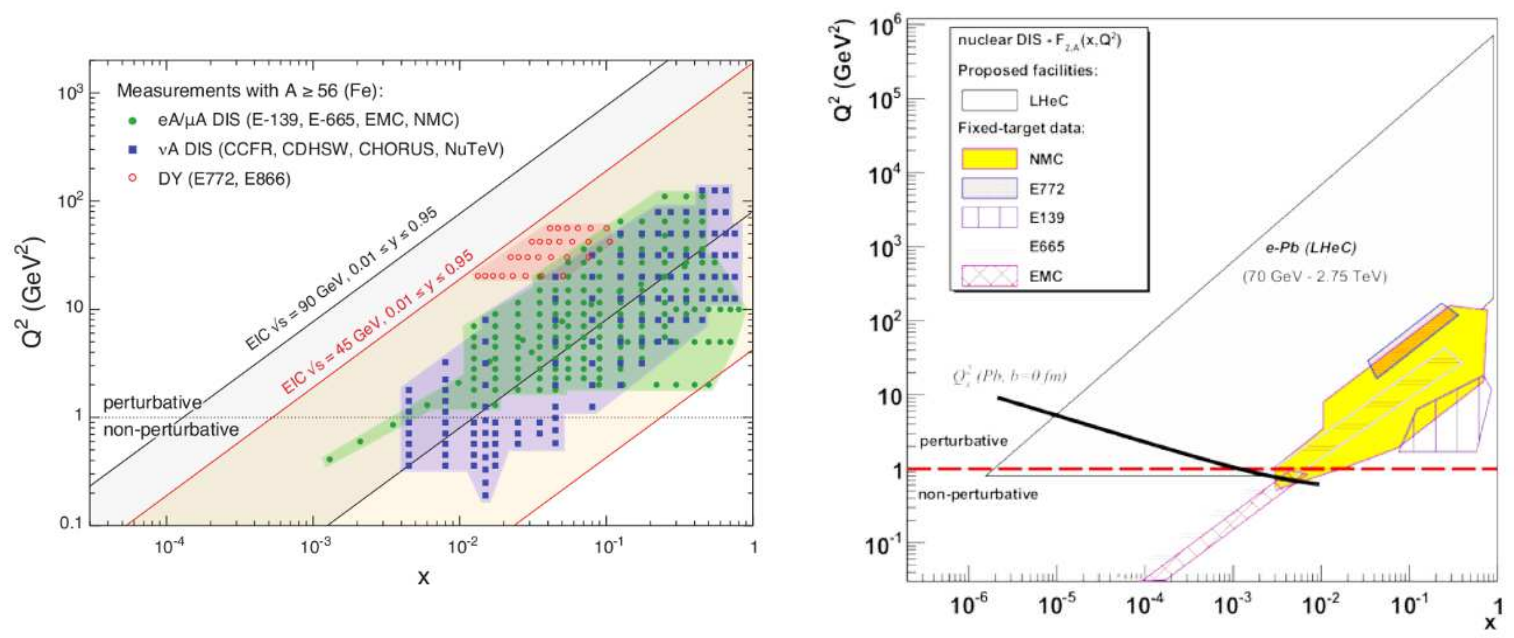

Figure 5: Leftmost panel: The kinematic range in $x$ and $Q^{2}$ of an EIC compared to fixed target charged-lepton and neutrino DIS, and Drell-Yan experiments, taken from [23]. Rightmost panel: The kinematic range in $x$ and $Q^{2}$ covered by the LHeC compared to fixed target charged-lepton DIS, and Drell-Yan experiments, taken from [24] .

The two structure functions, $F_{2}$ and $F_{L}$, in terms of which the differential cross-section of unpolarized DIS is usually written, can be measured at an EIC. In Fig. 6 a simulation of these observables versus $Q^{2}$ for different values of $x$ at an EIC is presented. In the leftmost panel of this figure, it is shown, by comparing the uncertainty bands of $F_{2}$ derived from the employment of EPS09 nPDFs (in gray) with the uncertainties of the data points, that an EIC would have a sizable impact on reducing the uncertainties for $x \leq 0.01$. The longitudinal structure function, shown in the rightmost panel of this figure, is very difficult to measure, but it is also a really interesting quantity due to its potential to constrain the gluons at low $x$. Furthermore, as it can be seen in Fig. 6, the kinematic extent of the $F_{2}$ is much wider than that of the $F_{L}$.

A completely (complementary) new proposal to study the nuclear gluon is the analysis of the charm structure function $F_{2}^{c \bar{c}}[23]$ - never measured before in e+A collisions. This is presented in Fig. 7. One more time, by comparing the uncertainty bands of $F_{2}^{c \bar{c}}$ derived from the employment of EPS09 nPDFs (in gray) with the uncertainties of the data points, one can see that an EIC may enable a considerable decrease of the uncertainties for $x \leq 0.005$. Other studies, where the inclusive and charm reduced cross section for an EIC are simulated using EPPS16 nPDFs, point in the same direction [25].

It is worth mentioning that there are analysis that quantify the effect that the $\mathrm{LHeC}$ data may 

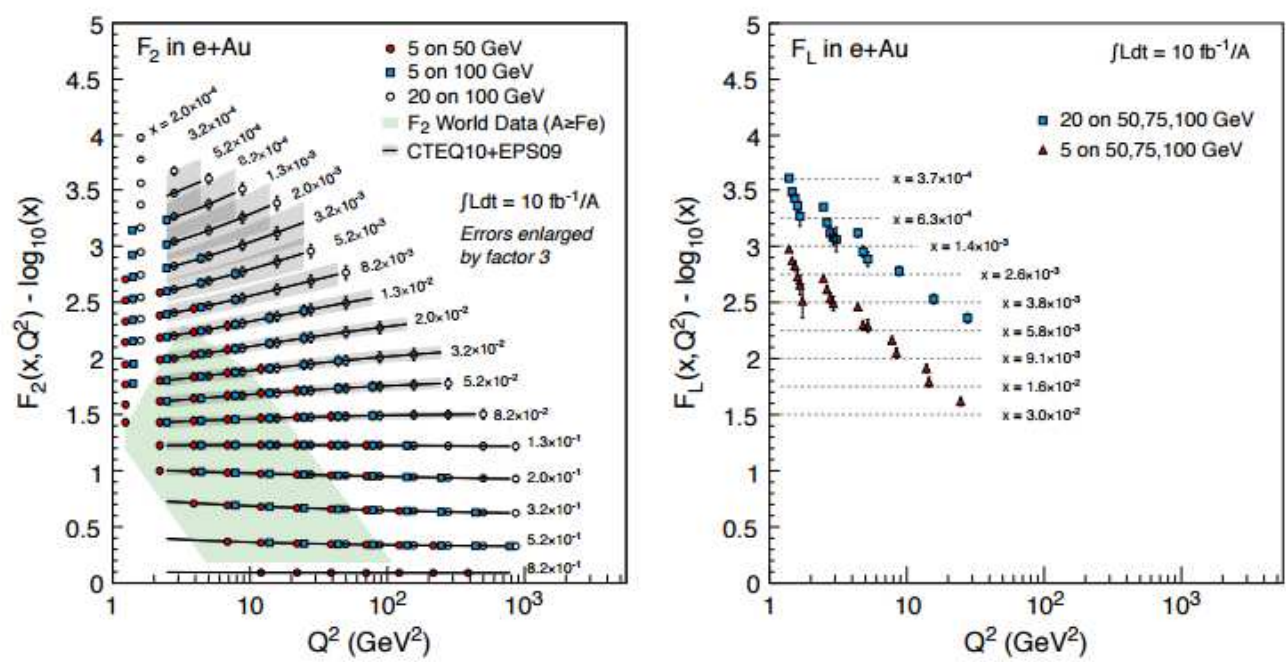

Figure 6: The structure functions $F_{2}$ (left panel) and $F_{L}$ (right panel) vs. $Q^{2}$ for several values of $x$ at an EIC generated by PYTHIA using EPS09 nPDFs. Figure taken from [23].

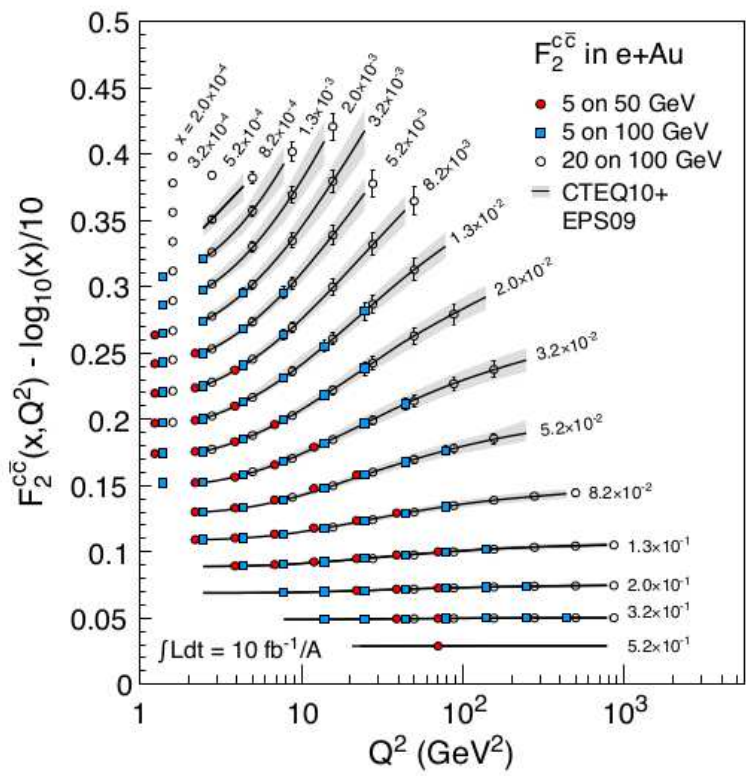

Figure 7: The charm structure functions $F_{2}^{c \bar{c}}$ vs. $Q^{2}$ for several values of $x$ at an EIC generated by PYTHIA using EPS09 nPDFs. Figure taken from [23]. 
have on nPDFs, as well. For instance, in Ref. [26] it is shown that this experiment would lead to a remarkable reduction of the small- $x$ uncertainties of the light sea quarks and gluons.

\section{Summary}

The current status of nuclear parton distribution functions has been reviewed, making special emphasis on the latest improvements; that is, the inclusion - for the first time - of the LHC $\mathrm{p}+\mathrm{Pb}$ Run-I data in a global fit of nPDFs (EPPS16). This has demonstrated the constraining power of these data. However, more data need to be implemented, particularlly to constrain the nuclear partonic structure at low $x$. To this end, new facilities, such as the Electron-Ion Collider (EIC) and the Large Hadron electron Collider ( $\mathrm{LHeC}$ ) become imperative.

\section{Acknowledgments}

This research was supported by the European Research Council grant HotLHC ERC-2011StG-279579; the People Programme (Marie Curie Actions) of the European Union's Seventh Framework Programme FP7/2007-2013/ under REA grant agreement \#318921 (NA); Ministerio de Ciencia e Innovación of Spain under project FPA2014-58293-C2-1-P and FEDER; Xunta de Galicia (Consellería de Educación) - the group is part of the Strategic Unit AGRUP2015/11. I acknowledge the Spanish Ministry of Education, Culture and Sports for financial support (grant FPU2013-03558).

\section{References}

[1] R. K. Ellis, H. Georgi, M. Machacek, H. D. Politzer and G. G. Ross, Nucl. Phys. B 152285 (1979).

[2] J. C. Collins, D. E. Soper and G. F. Sterman, Adv. Ser. Direct. High Energy Phys. 51 (1989).

[3] R. Sassot, M. Stratmann and P. Zurita, Phys. Rev. D 81054001 (2010).

[4] Yu. L. Dokshitzer, D. I. Dyakonov and S. I. Troyan, Phys. Report. 58269 (1980).

[5] G. Altarelli and G. Parisi, Nucl. Phys. B 126298 (1977).

[6] V. N. Gribov and L. N. Lipatov, Sov. J. Nucl. Phys. 15675 (1972) [Yad. Fiz. 151218 (1972)].

[7] G. P. Salam and J. Rojo, Comput. Phys. Commun. 180120 (2009).

[8] P. Santorelli and E. Scrimieri, Phys. Lett. B 459, 599 (1999).

[9] A. Cafarella, C. Coriano and M. Guzzi, Nucl. Phys. B 748253 (2006).

[10] J. J. Aubert et al. [European Muon Collaboration], Phys. Lett. 123B, 275 (1983).

[11] K. J. Eskola, H. Paukkunen and C. A. Salgado, JHEP 0904, 065 (2009).

[12] D. de Florian, R. Sassot, P. Zurita and M. Stratmann, Phys. Rev. D 85074028 (2012).

[13] H. Khanpour and S. Atashbar Tehrani, Phys. Rev. D 93 no.1, 014026 (2016).

[14] K. Kovarik et al., Phys. Rev. D 93, no. 8, 085037 (2016).

[15] K. J. Eskola, P. Paakkinen, H. Paukkunen and C. A. Salgado, Eur. Phys. J. C 77 no.3, 163 (2017). 
[16] H. Paukkunen, Nucl. Phys. A 92624 (2014).

[17] N. Armesto, J. Rojo, C. A. Salgado and P. Zurita, JHEP 1311015 (2013).

[18] N. Armesto, H. Paukkunen, J. M. Penín, C. A. Salgado and P. Zurita, Eur. Phys. J. C 76 no.4, 218 (2016).

[19] V. Khachatryan et al. [CMS Collaboration], Phys. Lett. B 750565 (2015).

[20] G. Aad et al. [ATLAS Collaboration], Phys. Rev. C 92 no.4, 044915 (2015).

[21] S. Chatrchyan et al. [CMS Collaboration], Eur. Phys. J. C 74, no. 7, 2951 (2014).

[22] F. Arleo and S. Peigné, Phys. Rev. D 95, no. 1, 011502 (2017).

[23] A. Accardi et al., Eur. Phys. J. A 52, no. 9, 268 (2016).

[24] J. L. Abelleira Fernandez et al. [LHeC Study Group], J. Phys. G 39, 075001 (2012).

[25] E. C. Aschenauer, S. Fazio, M. A. C. Lamont, H. Paukkunen and P. Zurita, Phys. Rev. D 96, no. 11, 114005 (2017).

[26] I. Helenius, H. Paukkunen and N. Armesto, PoS DIS 2016, 276 (2016). 